\title{
Lower Extremity Radicular Pain Caused by Entrapped Sigmoid Colon Between L5 and S1 Vertebrae
}

\author{
Sanghyung Ko, $\mathrm{MD}^{1}$, Noh Kyoung Park, $\mathrm{MD}^{1}$, Kyoung Jin Cho, $\mathrm{MD}^{1}$, Jung Hyun Baek, $\mathrm{MD}^{1}$, \\ Jeong-Wook Lim, $\mathrm{MD}^{2}$, Dongjin Choi, $\mathrm{MD}^{3}$, Sangkuk Kang, $\mathrm{MD}, \mathrm{PhD}^{1}$
}

Departments of ${ }^{1}$ Physical Medicine and Rehabilitation, ${ }^{2}$ Neurosurgery, and ${ }^{3}$ Surgery, Sun General Hospital, Daejeon, Korea

Intestinal entrapment between two vertebral bodies is very rare. In all previous cases, it occurred by major trauma. However, the bowel entrapment between two vertebral bodies without trauma has never been reported, not to mention as the cause of lower extremity radicular pain. We describe the case of an 82-year-old female patient with right lower extremity radicular pain without recent trauma history. The patient was diagnosed sigmoid colon entrapment between the L5 and S1 vertebrae by lumbar spinal computerized tomography and magnetic resonance imaging, and showed improvement in radicular pain after manual reduction of interpositioned colon during surgery. Intestinal entrapment between two vertebrae without trauma is caused by degenerative and vacuum changes of the intervertebral disc combined with the anterior longitudinal ligament injury.

Keywords Radiculopathy, Intervertebral disc, Colon entrapment

\section{INTRODUCTION}

Radicular pain in the lower extremity is a common symptom that occurs as a result of a herniated lumbar disc or lumbar spinal stenosis. There are also other etiologies of lower extremity radicular pain: trauma, intraspinal mass, infection, etc. It has never been reported, however, that the intestinal entrapment between two ver-

Received September 15, 2014; Accepted January 6, 2015

Corresponding author: Sangkuk Kang

Department of Physical Medicine and Rehabilitation, Sun General Hospital, 29 Mokjung-ro, Jung-gu, Daejeon 34811, Korea

Tel: +82-42-220-8468, Fax: +82-303-3130-8468, E-mail: rmactksk@daum. net

(c) This is an open-access article distributed under the terms of the Creative Commons Attribution Non-Commercial License (http://creativecommons. org/licenses/by-nc/4.0) which permits unrestricted noncommercial use, distribution, and reproduction in any medium, provided the original work is properly cited.

Copyright (C) 2015 by Korean Academy of Rehabilitation Medicine tebrae was the cause of lower extremity radicular pain. We report a case of lower extremity radicular pain that showed improvement after reduction of the interpositioned intestine between vertebral bodies.

\section{CASE REPORT}

An 82-year-old woman, who had experienced lumbago for 2 weeks, visited a local medical clinic with complaints of radiating pain in her right leg, nausea, and abdominal discomfort. She received a painkilling injection and oral medications, causing her abdominal symptoms to subside, but her leg pain was aggravated, which brought her to the emergency department.

Neurological examination revealed hypesthesia on the right S1 dermatome. Her leg pain scored $7-8 / 10$ on the Numeric Rating Scale (NRS). The straight leg raising test 
showed $40^{\circ}$ for the right leg and $80^{\circ}$ for the left leg. The manual muscle test (MMT) of the right lower extremity, using the Medical Research Council scale, revealed 4/5 hip flexors, $3 / 5$ hip extensors, $5 / 5$ knee extensors, $4 / 5$ ankle dorsiflexors, $3 / 5$ ankle plantar flexors, and $4 / 5$ great toe extensors.

In her medical history, the patient had a total hysterectomy for the treatment of uterine cervical cancer 30 years ago, and two partial small bowel resections for the

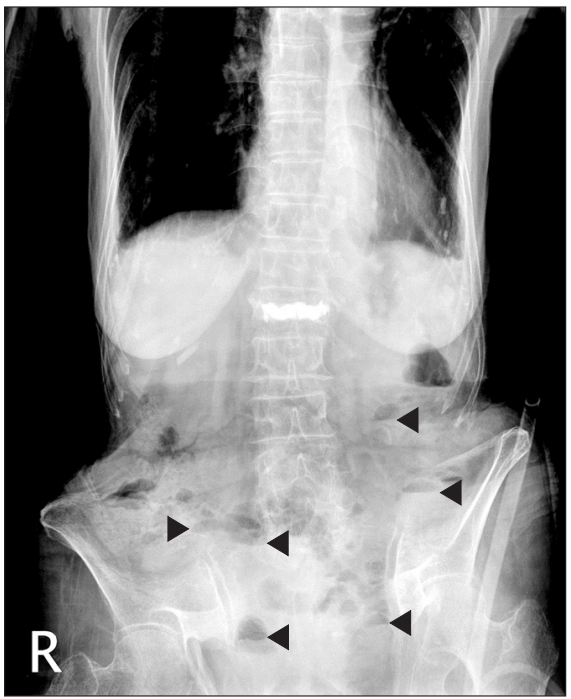

Fig. 1. Plain erect X-ray images of the abdomen show multiple air-fluid levels in the intestine (arrow head). treatment of ileus about 18 months and 6 months ago. Additionally, she had a percutaneous vertebroplasty 10 months ago due to a compression fracture of the first lumbar vertebral body after a slip. But, she had no recent remarkable trauma history.

On plain erect abdominal X-ray, multiple air-fluid levels were observed in the intestine (Fig. 1). Lumbar spinal computerized tomography (CT) showed an entrapped intestine within the intervertebral disc space between the L5 and S1 vertebrae (Fig. 2A, B). There was no significant fracture or dislocation of vertebrae. She was admitted in the Department of General Surgery and consulted to a neurosurgeon. Lumbar spinal magnetic resonance imaging (MRI) demonstrated discontinuity of the anterior longitudinal ligament (ALL) at the L5 and S1, disappearance of most of the L5-S1 intervertebral disc, and an entrapped intestine in the vacant intervertebral disc space. The remnant disc materials were pushed back, narrowing the spinal canal. In addition, a compressed right S1 nerve root and left facet joint destruction were found on the MR images (Fig. 3A, B). In other regions, L3-4 disc herniation and L4-5 disc bulging were shown, but nerve root compression was not found.

On the 4th day of admission, the patient underwent a combined operation by a general surgeon and a neurosurgeon. First, laparotomy was performed, and the entrapped sigmoid colon was found in intervertebral space between the L5 and S1 vertebrae. The sigmoid colon was
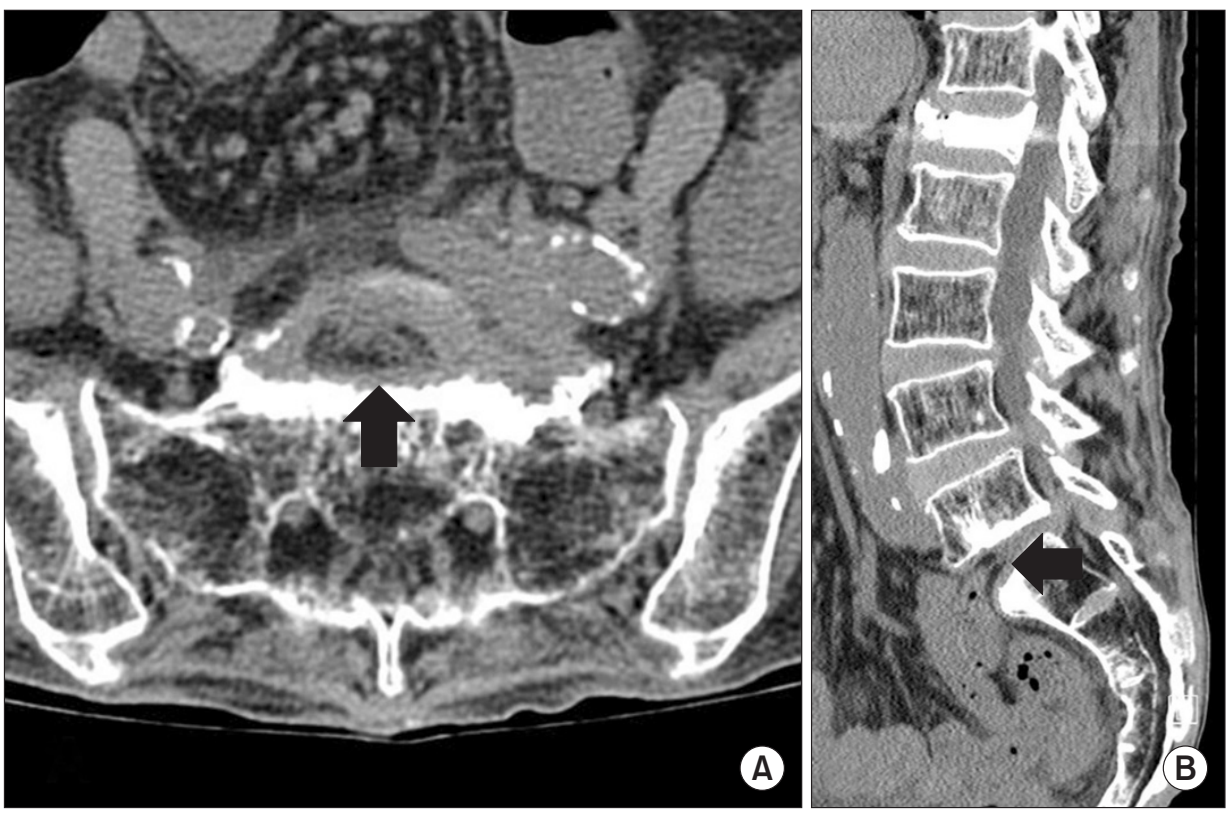

Fig. 2. (A) Axial and (B) sagittal Lspine computerized tomography images show the entrapped intestine in the L5 to S1 intervertebral space (arrow). 

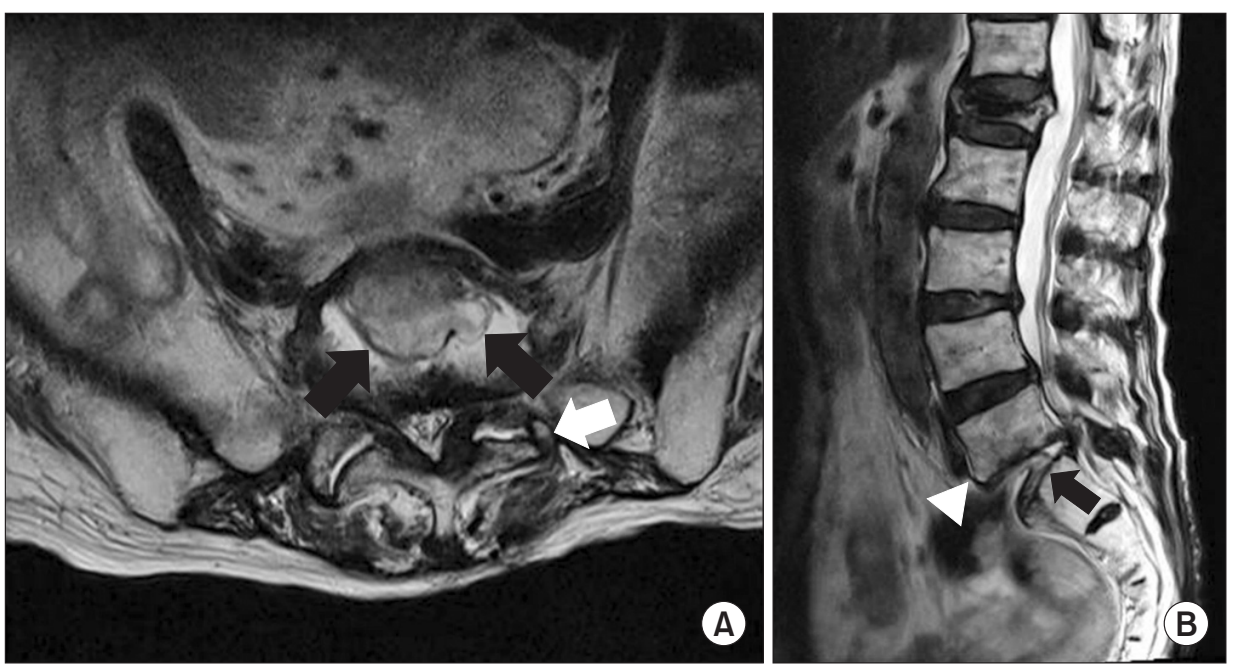

Fig. 3. (A) Axial and (B) sagittal T2-weighted MR images reveal the entrapped intestine in the L5 to S1 intervertebral space (black arrow), facet joint destruction (white arrow), and the non-contiguous ligament injury in the anterior longitudinal ligament at the disc level of L5-S1 (arrow head).

retrieved by manual reduction and did not have any necrosis. In the findings during surgery, the disc material between the L5 and S1 vertebrae remained only a small amount. Next, the remnant of the disc material was removed, then anterior interbody fusion was performed by the neurosurgeon in order to prevent a recurrence of colon entrapment in the L5-S1 intervertebral space. After surgery, the patient's right leg pain was much improved from $7-8 / 10$ to $2-3 / 10$ according to NRS. But, the weakness of the right lower extremity persisted, and the patient was transferred to the Department of Rehabilitation Medicine on the 15th post-operative day.

At the time of transfer, the patient's overall muscle strength had decreased due to bedrest after surgery. According to the MMT, muscle strength in both lower extremities was graded as $4 / 5$, except for the right hip extension and right ankle plantarflexion at $3 / 5$. Her radicular pain remained at $2-3 / 10$ on the NRS, mostly on the right $\mathrm{S} 1$ dermatome as a tingling sensation. On the functional evaluation, sitting and standing up could be performed independently, but minimal assistance was necessary for balanced-level walking with anterior walker. She received rehabilitation therapies including heat, electrical stimulation, strengthening exercise, and gait training. She gradually recovered the ability to walk about $50 \mathrm{~m}$ with a cane two weeks after rehabilitation, and was transferred to a convalescent hospital near her home by the request of her family.

\section{DISCUSSION}

Intestinal entrapment between two vertebrae is very rare. There have been seven cases of bowel entrapment between vertebral bodies reported in the literature [17]. In these cases, intestinal entrapment between vertebrae occurred by trauma such as a traffic accident, fall, or crash into a heavy object, and it was accompanied by fracture or dislocation of vertebrae. The mechanism of the entrapment is not known. The most reliable hypothesis is that hyperextension of the lumbar spine due to trauma causes the rupture of ALL and negative pressure of the intervertebral space that draws the bowel between two vertebral bodies $[1,2]$. In our case, however, the patient did not have recent trauma history, and the radiologic findings showed no significant fracture or dislocation on the L5 and S1 vertebrae.

Hwang and Park [8] reported a case of entrapped inferior vena cava between L4 and L5 vertebral bodies without a definite recent trauma. They supposed that it could occur under conditions of degeneration and vacuum changes of the intervertebral disc and widening of the intervertebral space due to already existing partial compression fractures of the intervening vertebral bodies. Also, the focal defect of adjacent ALL may be caused by hyperextension of the lumbar spine. It seems that our case demonstrated a similar mechanism for intestinal entrapment between vertebral bodies. The L5-S1 disc material was partially lost due to degenerative changes and the vacuum phenomenon as an aging process. The fading intervertebral disc gradually produced negative 
pressure within the intervertebral disc space. Additionally, the adjacent ALL got weaker due to the degenerative change and lumbar hyperextension, then was eventually torn. The defected ALL in our case was shown in the lumbar MRI (Fig. 3B). Because the ALL works as an important barrier between the intestine and the vertebrae, the injured ALL on the lesion drew the sigmoid colon into the intervertebral space by the negative pressure. But, unlike in the case of Hwang and Park [8], the compression fracture of intervening vertebral bodies was not combined in our case. The main reason for the injured ALL without compression fracture is not certain, but we suppose that it may relate to facet joint destruction. In accordance with Roaf's studies on mechanisms of spinal injuries [9], the ALL is very resistant to compression, distraction, flexion and extension, but it is vulnerable to rotation and horizontal shearing forces. As rotation is anatomically limited by the facet joint in a normal lumbosacral spine, it is not likely that rotating force is applied to the ALL without accompanying fracture or dislocation of vertebrae. In this patient, the facet joint of L5-S1 was severely destructed by the degenerative change (Fig. $3 \mathrm{~A}$ ), so it is assumed that repetitive rotating force could be applied to the ALL and cause damage to it. However, we cannot exclude the possibility of iatrogenic injury on the presacral fascia and ALL during the previous abdominal surgeries.

For this patient, the chief complaint was a radicular pain to the lower extremity, unlike previous reports, presenting the symptoms of ileus. This was because the adjacent colon was pulled to the intervertebral disc space through the defective ALL and pushed the degenerative disc backward to the vertebral canal. Fortunately, the entrapped colon did not have any necrosis nor did it need a bowel resection.

Although it is extremely rare to find the cause of radiating pain to be intestinal entrapment between vertebrae, it seems worthwhile to consider CT or MRI in the early phase for aged patients with spine trauma or abdominal surgery in their medical history if accompanied by abdominal pain, nausea or vomiting.

\section{CONFLICT OF INTEREST}

No potential conflict of interest relevant to this article was reported.

\section{REFERENCES}

1. Ford WD. Traumatic incarceration of the jejunum between two lumbar vertebrae. J Pediatr Surg 1979; 14:189-90.

2. Davis RE, Mittal SK, Perdikis G, Richards AT, Fitzgibons RJ Jr. Traumatic hyperextension/hyperflexion of the lumbar vertebrae with entrapment and strangulation of small bowel: case report. J Trauma 2000;49:958-9.

3. Metaizeau JP, Prévot J, Schmitt M, Bretagne MC. Intestinal strangulation between two vertebra following an axial dislocation of L1/L2. J Pediatr Surg 1980;15:193-4.

4. Rodger RM, Missiuna P, Ein S. Entrapment of bowel within a spinal fracture. J Pediatr Orthop 1991;11:783-5.

5. Silver SF, Nadel HR, Flodmark O. Pneumorrhachis after jejunal entrapment caused by a fracture dislocation of the lumbar spine. AJR Am J Roentgenol 1988;150:1129-30.

6. Vermassen F, Van Belleghem Y, Van Velthoven V, Derom F. Acute bowel entrapment between two lumbar vertebrae. Injury 1988;19:449-50.

7. Eldridge TJ, McFall TM, Peoples JB. Traumatic incarceration of the small bowel: case report. J Trauma 1993;35:960-1.

8. Hwang SS, Park SY. Entrapped inferior vena cava between 2 lumbar vertebrae. J Comput Assist Tomogr 2009;33:811-3.

9. Roaf R. A study of the mechanics of spinal injuries. J Bone Joint Surg Br 1960;42:810-23. 